\title{
Motion of charged particle in Reissner-Nordström spacetime: a Jacobi-metric approach
}

\author{
Praloy Das ${ }^{\mathrm{a}}$, Ripon $\mathrm{Sk}^{\mathrm{b}}$, Subir Ghosh ${ }^{\mathrm{c}}$ \\ Physics and Applied Mathematics Unit, Indian Statistical Institute, 203 B. T. Road, Kolkata 700108, India
}

Received: 13 June 2017 / Accepted: 10 October 2017 / Published online: 3 November 2017

(C) The Author(s) 2017. This article is an open access publication

\begin{abstract}
The present work discusses motion of neutral and charged particles in Reissner-Nordström spacetime. The constant energy paths are derived in a variational principle framework using the Jacobi metric which is parameterized by conserved particle energy. Of particular interest is the case of particle charge and Reissner-Nordström black hole charge being of same sign, since this leads to a clash of opposing forces-gravitational (attractive) and Coulomb (repulsive). Our paper aims to complement the recent work of Pugliese et al. (Eur Phys J C 77:206. arXiv:1304.2940, 2017; Phys Rev D 88:024042. arXiv:1303.6250, 2013). The energy dependent Gaussian curvature (induced by the Jacobi metric) plays an important role in classifying the trajectories.
\end{abstract}

\section{Introduction}

Point particle dynamics serves the important purpose of initiating the study of a more complex motion of extended bodies. A major area of interest, especially in recent times, is the motion of particles in the presence of black holes and naked singularities. The exhaustive topical work by Pugliese et al. $[1,2]$ (as well as their series of earlier papers [3-6]) have revealed clearly the possibility of distinguishing between black holes and naked singularities in the case of charged particle dynamics in Reissner-Nordström background. The systematic analysis is based on the nature of particle motion for different charge to mass ratio in the Reissner-Nordström metric, (which is expected), but the non-trivial effect of the charge to mass ratio of the probe particle is indeed a surprising element. In this perspective, the aim of the present paper, dealing once again with the motion of a point charge in the

\footnotetext{
a e-mail: praloydasdurgapur@gmail.com

b e-mail: riponphysics@gmail.com

c e-mail: subirghosh20@gmail.com
}

presence of a charged black hole, is not to add any more details to the results of $[1,2,7]$, but to complement this reference's observations and bring in new insights from an entirely different (and hitherto less explored) point of view, that of the Jacobi-metric approach. This framework has generated a lot of interest after the series of publications by Gibbons [8] and by Chanda et al. $[9,10]$.

The primary major difference is the following. On the one hand, Pugliese et al. [1-3] rely on the covariant framework where geodesic motion takes place in the ReissnerNordström spacetime. The Lagrangian equation of motion is derived from the unrestricted variational principle with the charge and mass parameters of the source and probe dictating the particle motion for arbitrary particle energy, which is a derived quantity. On the other hand, in a Jacobi-metric framework, the equation of motion is obtained from a restricted variational principle with fixed energy where the Lagrangian depends on the Jacobi metric, which involves the particle energy explicitly. Schematically for a system with $E=K-V$ where $E, K, V$ are total energy, kinetic energy and potential energy of a probe particle, respectively, the Jacobi metric is given by

$j=2(E-V) g$

where $j$ is the Jacobi metric and $g$ the Riemannian metric on the manifold where the motion is taking place. In a sense each particle is performing a geodesic motion in its own space (time). The Jacobi metric is manifestly non-covariant (on a spatial slice) $[8,9]$ and the equation of motion is derived from a restricted (Maupertuis) variational principle with fixed particle energy. Hence the energy acts as an additional parameter along with the charge and mass parameters. The particle motion is still geodesic in the Jacobi-metric space. (The fact that the Jacobi metric is degenerate at the boundary where $E=V$ can create complications. For a detailed analysis of this issue and of ways of overcoming it, see for example [11].) 
A novelty in our analysis is the associated (Gaussian) curvature corresponding to the Jacobi metric, in line with [8]. In the next section we will see that even dynamics in flat space can give rise to an effective non-zero curvature from Jacobimetric space. In fact trajectories can be classified according to the sign of the curvature: positive, negative and zero curvature (for Jacobi metric) corresponds to elliptic (with negative energy), hyperbolic (positive energy) and parabolic (zero energy) orbits, respectively.

Apart from the importance of curvature in the Jacobi metric, one way in which the present work can complement the exhaustive analysis of $[1,2]$ is the following: whereas $[1,2]$ relies on an extremely detailed graphical analysis of the orbit structure keeping exact expressions we have provided analytic expressions of the orbits, albeit in a perturbative framework (of small charge of both the black hole and the probe). Also, to be compared to $[1,2]$, we have not restricted the analysis to circular orbits only.

We conclude this brief introduction by presenting the structure of the present work. The paper is organized as follows: In Sect. 2 we discuss generalities related to the Maupertuis principle and Jacobi metric as well as its application in problems of particle dynamics in black hole spacetimes. Section 3 deals with the neutral massive particle motion in Reissner-Nordström background. Section 4 constitutes the study of motion of charged massive particle in ReissnerNordström background. In Sect. 5 we provide a detailed analysis of Gaussian curvature for Jacobi metrics in the cases we study. The paper ends with our conclusions in Sect. 6. Detailed results are given Appendices A and B.

\section{Maupertuis principle and Jacobi metric}

The Maupertuis transformation and the associated Jacobimetric approach has generated a lot of recent interest $[8,9]$. To understand the context let us start by considering the obvious fact that a curve can be parameterized in an infinite number of ways (see for example [12] for a detailed discussion). For example, an ellipse

$\frac{x^{2}}{a^{2}}+\frac{y^{2}}{b^{2}}=1$

can be parameterized by

$x=a \sin (t), \quad y=b \cos (t)$,

and also by a different parameter $\tilde{t}$ related to $t$ by

$\tilde{t}=\left(a^{2}+b^{2}\right) t+\left(a^{2}-b^{2}\right) \sin (t)$.

Clearly the former parameterization (3) is connected to a two dimensional oscillator whereas the latter (4) is related to the Kepler problem. Generically the same trajectory can be attributed to distinct integrable systems where the parameter $t$ plays the role of time variable, conjugate to the respective Hamiltonian. Hence the question is how to derive the particular parameterization that matches with a known or interesting problem. The Maupertuis principle recasts this problem in a dynamical setup. The Maupertuis variational principle states that, given an $n$-dimensional configuration space Lagrangian $L\left(q_{i}, \dot{q}_{i}\right)$, the extremals of the action

$S=\int \mathrm{d} t L\left(q_{i}, \dot{q}_{i}\right)$

coincide with extremals of the reduced action in a $2 n-1$ dimensional phase space that is a level set of the Hamiltonian function $H\left(q_{i}, p_{i}\right)=E$. Here $p_{i}$ constitute the conjugate momenta to $q_{i}$ and the variation takes place on a fixed energy $(E)$ hypersurface. In a more explicit way let us suppose a natural Hamiltonian in a Riemanniam manifold with metric $g_{i j}$ to hold,

$H(q, p)=T(q, p)+V(q)=g_{i j}(q) p_{i} p_{j}+V(q)$

The trajectories for $H$ on a fixed energy smooth submanifold will coincide with trajectories of a new Hamiltonian $\tilde{H}$ given by

$\tilde{H}(q, p)=\tilde{T}(q, p)=\frac{g_{i j}(q)}{E-V(q)} p_{i} p_{j}$,

along with a transformation of the parameters,

$\mathrm{d} \tilde{t}=(E-V(q)) \mathrm{d} t$.

Together $(7,8)$ constitute the Jacobi transformation. A scaling of the effective metric provides the Jacobi metric. The two major novelties and advantages of the Maupertuis-Jacobi framework are:

1. The Hamiltonian $\tilde{H}$ yields a geodesic motion. This allows one to treat a dynamical problem as a geodesic motion and the well-known machinery of the geodesic motion can be directly exploited.

2. The Maupertuis principle and subsequent Jacobi transformation preserve integrability, that is, under this map an integrable system with a natural Hamiltonian goes over to another integrable system in the same phase space. Thus this scheme provides a method to search for new integrable systems.

3. The Gaussian curvature induced by the Jacobi metric allows one to classify the trajectories based on their energy since it appears explicitly in Jacobi metric. 
For a generic Lagrangian of the form (following the notation of [8])

$L=\frac{1}{2} m_{i j}(x) \dot{x^{i}} \dot{x^{j}}-V(x)$

it was shown by Jacobi that the constrained motion of a particle with energy $E$ is provided by geodesics of the rescaled metric

$j_{i j} \mathrm{~d} x^{i} \mathrm{~d} x^{j}=2(E-V) m_{i j} \mathrm{~d} x^{i} \mathrm{~d} x^{j}$.

It is interesting to observe that particle interactions can induce a curvature in the Jacobi metric through the potential function in an otherwise flat Newtonian space. One of the early workers on this topic was Pin [13] who considered many body systems and in particular showed that the Gaussian curvature of the Jacobi metric has a sign opposite to the particle energy $E$ (non-relativistic, without the rest energy). In later times Gibbons et al. [14-17] have considered the optical metric in various physical situations which is a closely related concept for massless particles. Discussions of the Jacobi-metric approach in a modern perspective can be found in [18].

Again very recently the Jacobi-metric formalism in a relativistic scenario has been applied by Gibbons [8] in an elegant study of massive particle motion in Schwarzschild spacetime. In the present paper we closely follow and extend this work to massive particle motion in a Reissner-Nordström background. Indeed, the Jacobi metric for a more general metric, that is, the Kerr-Newman metric, has been derived in $[9,10]$, although the probe particle dynamics was not considered there. We consider both cases of the probe particle being neutral and charged. The results show a qualitative difference between the two cases since in the latter one needs to consider the additional Coulomb interaction term between the source and probe particle. For a neutral particle the correction terms depend on $Q^{2}, Q$ being the charge of the black hole but interestingly for the charged probe correction terms involve $q Q$ terms as well, $q$ being charge of the particle, showing that the relative signs of the particle and the black hole charge become important.

For the generic metric

$\mathrm{d} s^{2}=-V^{2} \mathrm{~d} t^{2}+g_{i j} \mathrm{~d} x^{i} \mathrm{~d} x^{j}$

the action for a massive particle in this background can be written as

$S=-m \int L \mathrm{~d} t=-m \int \mathrm{d} t \sqrt{V^{2}-g_{i j} \dot{x^{i}} \dot{x^{j}}}$.

The canonical momentum

$$
p_{i}=\frac{m \dot{x}^{i}}{\sqrt{V^{2}-g_{i j} \dot{x^{i} \dot{x}^{j}}}}
$$

leads to the Hamiltonian

$$
H=\sqrt{m^{2} V^{2}+V^{2} g^{i j} p_{i} p_{j}} .
$$

This yields the Hamilton-Jacobi equation for the geodesics, parameterized by the energy $E$,

$\sqrt{m^{2} V^{2}+V^{2} g^{i j} \partial^{i} S \partial_{j} S}=E$

where $p_{i}=\partial_{i} S$. Finally, the Hamiltonian-Jacobi equation for geodesics of the Jacobi metric $j_{i j}$ is given by

$\frac{1}{E^{2}-m^{2} V^{2}} f^{i j} \partial_{i} S \partial_{j} S=1$

where $j_{i j}$ is defined as

$j_{i j} \mathrm{~d} x^{i} \mathrm{~d} x^{j}=\left(E^{2}-m^{2} V^{2}\right) V^{-2} g_{i j} \mathrm{~d} x^{i} \mathrm{~d} x^{j}$.

In fact, $f_{i j}=V^{-2} g_{i j}$ turns out to be the optical or Fermat metric. For massless particles $(m=0)$ the Jacobi metric becomes equal to the Fermat metric modulo a factor of $E^{2}$ and subsequently the geodesics do not depend upon energy E. However, in the massive case, $m \neq 0$, the geodesics are E-dependent.

Let us put our work in its proper perspective. The explicit results and observations of the present work are not entirely new. Some of these are discussed in the book by Chandrasekhar [19]. More recent and detailed studies are provided in $[1-3,20]$. However, we have revisited the system from the Jacobi-metric point of view. The Jacobi-metric construction for charged massive particle and the subsequent analysis is completely new. Furthermore in the present work the Gaussian curvature induced by the Jacobi metric plays an essential role since the motion is geodesic in nature. We have compared results computed from Gaussian curvature consideration (in a Jacobi-metric approach) with similar results obtained via conventional scheme. For the charged probe, the interplay between the gravitational and Coulomb forces proves to be interesting.

\section{Jacobi metric for neutral particle in Reissner-Nordström Geometry}

The Reissner-Nordström metric is a spherically symmetric solution of the coupled Maxwell Einstein gravity. It represents a black hole with mass $M$ and charge $Q$ and is given by

$$
\begin{aligned}
\mathrm{d} s^{2}= & -\left(1-\frac{2 M}{r}+\frac{Q^{2}}{r^{2}}\right) c^{2} \mathrm{~d} t^{2} \\
& +\frac{\mathrm{d} r^{2}}{\left(1-\frac{2 M}{r}+\frac{Q^{2}}{r^{2}}\right)}+r^{2} \mathrm{~d} \theta^{2}+r^{2} \sin ^{2} \theta \mathrm{d} \phi^{2} .
\end{aligned}
$$

For $Q=0$ the Schwarzschild metric is recovered. As is well known it has two horizons, at $r=r_{ \pm}=M \pm \sqrt{M^{2}-Q^{2}}$. The 
nature of the horizon singularities is different in ReissnerNordström and Schwarzschild geometries. The latter is spacelike whereas the former is timelike and thus yielding richer possibilities regarding the nature of trajectories. There are timelike worldlines for particles that can cross the $r_{+}$ horizon and skirting the singularity can move out to another spacetime region after crossing $r_{-}$. On the contrary for the Schwarzschild geometry, after crossing the event horizon at $r=2 M$ the particle has no option but to fall towards the singularity. The $r_{+}$-horizon acts like the $r=2 M$ event horizon of Schwarzschild; the $r_{-}$-horizon is termed the Cauchy horizon.

Now, generalizing the result of Gibbons [8], the Jacobi metric corresponding to the Reissner-Nordström solution is

$$
\begin{aligned}
\mathrm{d} s^{2}= & \left(E^{2}-m^{2}+\frac{2 M m^{2}}{r}-\frac{Q^{2} m^{2}}{r^{2}}\right) \\
& \times\left[\frac{\mathrm{d}^{2}}{\left(1-\frac{2 M}{r}+\frac{Q^{2}}{r^{2}}\right)^{2}}+\frac{r^{2} \mathrm{~d} \theta^{2}+r^{2} \sin ^{2} \theta \mathrm{d} \phi^{2}}{\left(1-\frac{2 M}{r}+\frac{Q^{2}}{r^{2}}\right)}\right] .
\end{aligned}
$$

The first part is the conformal factor, whereas the second factor is the optical metric. Due to spherical symmetry, we are allowed to study the system in the equatorial plane $\theta=\frac{\pi}{2}$ without loss of generality. This reduces the Jacobi metric to an expression of the form

$$
\begin{aligned}
\mathrm{d} s^{2}= & \left(E^{2}-m^{2}+\frac{2 M m^{2}}{r}-\frac{Q^{2} m^{2}}{r^{2}}\right) \\
& \times\left[\frac{\mathrm{d}^{2}}{\left(1-\frac{2 M}{r}+\frac{Q^{2}}{r^{2}}\right)^{2}}+\frac{r^{2} \mathrm{~d} \phi^{2}}{\left(1-\frac{2 M}{r}+\frac{Q^{2}}{r^{2}}\right)}\right] .
\end{aligned}
$$

Because of the axial symmetry $\phi$ is a cyclic coordinate so that the angular momentum $l$ is conserved,

$$
\begin{aligned}
l & =\left(E^{2}-m^{2}+\frac{2 M m^{2}}{r}-\frac{Q^{2} m^{2}}{r^{2}}\right)\left(\frac{r^{2}}{\left(1-\frac{2 M}{r}+\frac{Q^{2}}{r^{2}}\right)}\right)\left(\frac{\mathrm{d} \phi}{\mathrm{d} s}\right) \\
& =\text { constant. }
\end{aligned}
$$

Now, together with (20), (21) yields

$$
\begin{aligned}
& \left(E^{2}-m^{2}+\frac{2 M m^{2}}{r}-\frac{Q^{2} m^{2}}{r^{2}}\right) \\
& \times\left[\frac{1}{\left(1-\frac{2 M}{r}+\frac{Q^{2}}{r^{2}}\right)^{2}}\left(\frac{\mathrm{d}}{\mathrm{d} s}\right)^{2}\right. \\
& \left.+\frac{r^{2}}{\left(1-\frac{2 M}{r}+\frac{Q^{2}}{r^{2}}\right)}\left(\frac{\mathrm{d} \phi}{\mathrm{d} s}\right)^{2}\right]=1,
\end{aligned}
$$

which can be rewritten as

$$
\begin{gathered}
\left(E^{2}-m^{2}+\frac{2 M m^{2}}{r}\right. \\
\left.-\frac{Q^{2} m^{2}}{r^{2}}\right)^{2} \frac{1}{\left(1-\frac{2 M}{r}+\frac{Q^{2}}{r^{2}}\right)^{2}}\left(\frac{\mathrm{d}}{\mathrm{d} s}\right)^{2}=E^{2} \\
\quad-\left(1-\frac{2 M}{r}+\frac{Q^{2}}{r^{2}}\right)\left(m^{2}+\frac{l^{2}}{r^{2}}\right)
\end{gathered}
$$

This satisfies the standard result,

$m^{2}\left(\frac{\mathrm{d}}{\mathrm{d} \tau}\right)^{2}=E^{2}-\left(1-\frac{2 M}{r}+\frac{Q^{2}}{r^{2}}\right)\left(m^{2}+\frac{l^{2}}{r^{2}}\right)$.

Here $\tau$ is the proper time along the geodesic and the angular momentum is

$l=m r^{2}\left(\frac{\mathrm{d} \phi}{\mathrm{d} \tau}\right)$

for

$\mathrm{d} \tau=m \frac{\left(1-\frac{2 M}{r}+\frac{Q^{2}}{r^{2}}\right)}{\left(E^{2}-m^{2}+\frac{2 M m^{2}}{r}-\frac{Q^{2} m^{2}}{r^{2}}\right)} \mathrm{d} s$.

Incidentally the above relation connects the proper time $\tau$ to the Jacobi path length $s$. Conventionally the trajectory is expressed in terms of a new variable $u=\frac{1}{r}$ :

$\frac{\mathrm{d}^{2} u}{\mathrm{~d} \phi^{2}}+u=\frac{F(u)}{h^{2} u^{2}}$

where, for the Reissner-Nordström case, we find

$\frac{F(u)}{h^{2} u^{2}}=3 M u^{2}+\frac{M}{h^{2}}-2 Q^{2} u^{3}-\frac{Q^{2}}{h^{2}} u$.

In the above $h=l / m$ is the conserved angular momentum per unit mass. Thus (27) and (28) constitute the particle worldline or trajectory equation.

It is straightforward to solve (27) and generate the following first order differential equation:

$$
\begin{aligned}
\left(\frac{\mathrm{d} u}{\mathrm{~d} \phi}\right)^{2} & =-Q^{2} u^{4}+2 M u^{3}-u^{2}\left(1+\frac{Q^{2}}{h^{2}}\right)+\frac{2 M}{h^{2}} u+C \\
& =-Q^{2}(u-\alpha)(u-\beta)(u-\gamma)(u-\delta)
\end{aligned}
$$

where $\mathrm{C}$ is a constant related to the energy per unit mass $\in=\frac{E}{m}$ by

$C=\frac{\epsilon^{2}-1}{h^{2}}$.

This constitutes the first integral of motion and is one of our major results. Incidentally $\frac{h}{\epsilon}$ is the impact parameter. We immediately notice a qualitative change the black hole charge has brought about in the trajectory. In comparison 
to the Schwarzschild case [8] the present result involves a quartic term in $u$. Writing the quartic polynomial in terms of roots,

$$
\left(\frac{\mathrm{d} u}{\mathrm{~d} \phi}\right)^{2}=-Q^{2}(u-\alpha)(u-\beta)(u-\gamma)(u-\delta),
$$

the following identities are recovered:

$$
\begin{aligned}
& \alpha+\beta+\gamma+\delta=\frac{2 M}{Q^{2}}, \\
& \alpha \beta+\beta \gamma+\gamma \delta+\alpha \gamma+\alpha \delta+\beta \delta=\frac{1+\frac{Q^{2}}{h^{2}}}{Q^{2}},
\end{aligned}
$$

$\alpha \beta \gamma \delta=-\frac{C}{Q^{2}}$,

$\alpha \beta \gamma+\beta \gamma \delta+\gamma \delta \alpha+\alpha \beta \delta=\frac{2 M}{Q^{2}} h^{2}$.

Clearly these are extensions of analogous relations given in [8] for the Schwarzschild metric.

Our aim is to propose explicit solutions for the trajectories in the same manner as those derived in [8]. To that end let us quickly recapitulate the orbit equation for the Schwarzschild black hole [8,21],

$$
\left(\frac{\mathrm{d} u_{G}}{\mathrm{~d} \phi}\right)^{2}=2 M u_{G}^{3}-u_{G}^{2}+\frac{2 M}{h^{2}} u_{G}+C_{G},
$$

with the explicit solution

$u_{G}=A_{G}+\frac{B_{G}}{\cosh ^{2}\left(\omega_{G} \phi\right)}$.

The constant parameters $A_{G}, B_{g}, C_{G}, \omega_{G}$ are given in the appendix, with the subscript $G$ standing for the Gibbons solution [8].

Let us now come to our work. An explicit solution for the trajectory equation for the Reissner-Nordström case (29), studied here, is given by

$u=A+\frac{B}{\cosh ^{2}(\omega \phi)}+Q^{2} \frac{k}{\cosh ^{4}(\omega \phi)}$

where $A, B, k$ all are constants. One can solve for the constants perturbatively for small $Q$ to first non-trivial order in $Q^{2}$. The result is given in Appendix A. This constitutes one of our new results. This is a generic form of a closed orbit. As we have pointed out in the Introduction, this type of analytic result for a generic orbit, indeed in an approximate sense of a small charge $Q$ on the black hole, can complement the graphical analysis of $[1,2]$ without any approximations.

It is straightforward to reduce our analysis to circular orbit orbits for which $u=u_{c}$, a constant. We rewrite (29) as

$$
\begin{aligned}
\left(\frac{\mathrm{d} u}{\mathrm{~d} \phi}\right)^{2} & =-Q^{2} u^{4}+2 M u^{3}-u^{2}\left(1+\frac{Q^{2}}{h^{2}}\right)+\frac{2 M}{h^{2}} u+\frac{\epsilon^{2}-1}{h^{2}} \\
& \equiv f(u) .
\end{aligned}
$$

The presence of a biquadratic term of $u$ in $f(u)$, compared to a cubic one in the Schwarzschild geometry, is the qualitative change that leads to a significant difference only for the orbits that cross the event horizon at $r_{+}$and can skirt the singularity and come out of $r=r_{+}$, (as discussed earlier), instead of terminating at the singularity at $r=0$ as in the case of Schwarzschild black hole (see for example [19] for details).

For the occurrence of circular orbits the conditions are as follows:

$$
\begin{aligned}
f(u)= & -Q^{2} u^{4}+2 M u^{3}-u^{2}\left(1+\frac{Q^{2}}{h^{2}}\right) \\
& +\frac{2 M}{h^{2}} u+\frac{\epsilon^{2}-1}{h^{2}}=0, \\
f^{\prime}(u)= & -4 Q^{2} u^{3}+6 M u^{2}-2 u\left(1+\frac{Q^{2}}{h^{2}}\right) \\
& +\frac{2 M}{h^{2}}=0 .
\end{aligned}
$$

The relations change for the null geodesics which we are not considering at present (see for example [19]). We can easily obtain the expressions for the energy and angular momentum of a circular orbit of radius $r_{c}=\frac{1}{u_{c}}$ from the above two equations. The expressions are

$\epsilon^{2}=\frac{\left(1-2 M u_{c}+Q^{2} u_{c}^{2}\right)^{2}}{1-3 M u_{c}+2 Q^{2} u_{c}^{2}}$

and

$h^{2}=\frac{M-Q^{2} u_{c}}{u_{c}\left(1-3 M u_{c}+2 Q^{2} u_{c}^{2}\right)}$.

Incidentally, these results agree with [1,2].

The minimum radius for a stable circular orbit will occur at the point of inflection of the function $f(u)$, i.e.,

$f^{\prime \prime}(u)=-12 Q^{2} u^{2}+12 M u-2\left(1+\frac{Q^{2}}{h^{2}}\right)=0$.

Eliminating $h^{2}$ from the above equation using (41) we obtain

$4 Q^{4} u_{c}^{3}-9 M Q^{2} u_{c}^{2}+6 M^{2} u_{c}-M=0$,

or, in terms of $r_{c}$,

$r_{c}^{3}-6 M r_{c}^{2}+9 Q^{2} r_{c}-\frac{4 Q^{4}}{M}=0$

From (44), for $Q^{2}=0$, we recover the well-known result for the Schwarzschild geometry, $r_{c}=6 M$. However, neglecting $Q^{4}$, a leading order correction to the radius is easily obtained,

$r_{c} \approx 3 M \pm 3 M\left(1-\frac{Q^{2}}{M^{2}}\right)^{1 / 2} \approx 6 M-\frac{3}{2} \frac{Q^{2}}{M}$. 
Using Eqs. (38), (39) and (42), (37) takes the form

$\left(\frac{\mathrm{d} u}{\mathrm{~d} \phi}\right)^{2}=\left(u-u_{c}\right)^{3}\left(2 M-3 Q^{2} u_{c}-Q^{2} u\right)$,

and the solution is given by

$u=u_{c}+\frac{2\left(M-2 Q^{2} u_{c}\right)}{\left(M-2 Q^{2} u_{c}\right)^{2}\left(\phi-\phi_{0}\right)^{2}+Q^{2}}$.

For the Reissner-Nordström case, to $O\left(\frac{Q^{2}}{M}\right)$ we obtain

$r_{c}=6 M-\frac{3 Q^{2}}{2 M}$.

This constitutes the first part of our work.

\section{Jacobi metric for charged particle in Reissner-Nordström Geometry}

The next level of generalization is to consider the trajectory of a probe with charge $q$ in the presence of a charged black hole. Indeed this is a non-trivial extension to the previous case since an additional Coulomb interaction term of the form $\sim$ $(q Q) / r$ is involved. In Reissner-Nordström geometry where a test particle has a charge per unit mass $q$, the only nonvanishing component of the vector potential is $A_{0}$ and its motion is determined by the Lagrangian as of the form

$$
\begin{aligned}
2 L= & {\left[\left(1-\frac{2 M}{r}+\frac{Q^{2}}{r^{2}}\right)\left(\frac{\mathrm{d} t}{\mathrm{~d} \tau}\right)^{2}-\frac{1}{\left(1-\frac{2 M}{r}+\frac{Q^{2}}{r^{2}}\right)}\left(\frac{\mathrm{d}}{\mathrm{d} \tau}\right)^{2}\right.} \\
& \left.-r^{2}\left(\frac{\mathrm{d} \theta}{\mathrm{d} \tau}\right)^{2}-r^{2}\left(\sin ^{2} \theta\right)\left(\frac{\mathrm{d} \phi}{\mathrm{d} \tau}\right)^{2}\right]+2 \frac{q Q}{r} \frac{\mathrm{d} t}{\mathrm{~d} \tau} .
\end{aligned}
$$

However, we need not attempt to construct a generalized Reissner-Nordström solution starting from the EinsteinMaxwell point charge action. The Jacobi-metric formalism provides a quick answer. Thus for the Reissner-Nordström case with a charged probe, the Jacobi metric is given by

$$
\begin{aligned}
\mathrm{d} s^{2}= & \left(\left(E-\frac{m q Q}{r}\right)^{2}-m^{2}+\frac{2 M m^{2}}{r}-\frac{Q^{2} m^{2}}{r^{2}}\right) \\
& \times\left[\frac{\mathrm{d}^{2}}{\left(1-\frac{2 M}{r}+\frac{Q^{2}}{r^{2}}\right)^{2}}+\frac{r^{2} \mathrm{~d} \theta^{2}+r^{2} \sin ^{2} \theta \mathrm{d} \phi^{2}}{\left(1-\frac{2 M}{r}+\frac{Q^{2}}{r^{2}}\right)}\right] .
\end{aligned}
$$

The only distinct feature which arises due to the probe charge $q$ in this case is that the energy for a particle (having a turning point) that arrives at the event horizon will be $E=$ $\frac{m q Q}{r_{+}}$and this can be negative if $q Q<0$, which gives rise to the theoretical speculation of a generating energy from a black hole. Once again a restriction of the motion to the equatorial plane, i.e. $\theta=\frac{\pi}{2}$, reduces the Jacobi metric to

$$
\begin{aligned}
\mathrm{d} s^{2}= & \left(\left(E-\frac{m q Q}{r}\right)^{2}-m^{2}+\frac{2 M m^{2}}{r}-\frac{Q^{2} m^{2}}{r^{2}}\right) \\
& \times\left[\frac{\mathrm{d}^{2}}{\left(1-\frac{2 M}{r}+\frac{Q^{2}}{r^{2}}\right)^{2}}+\frac{r^{2} \mathrm{~d} \phi^{2}}{\left(1-\frac{2 M}{r}+\frac{Q^{2}}{r^{2}}\right)}\right] .
\end{aligned}
$$

In an identical fashion to the previous cases, we derive the trajectory equation for $u=\frac{1}{r}$,

$$
\begin{aligned}
\left(\frac{\mathrm{d} u}{\mathrm{~d} \phi}\right)^{2}= & -Q^{2} u^{4}+2 M u^{3}-u^{2}\left(1+\frac{Q^{2}}{h^{2}}-\frac{Q^{2} q^{2}}{h^{2}}\right) \\
& +\frac{2}{h^{2}}\left(M-\frac{q Q E}{m}\right) u+C
\end{aligned}
$$

where $h=l / m$ is the conserved angular momentum per unit mass, $C$ is a constant related to the energy per unit mass $\in=\frac{E}{m}$ and the angular momentum per unit mass $h$ occurs in the relation $C=\frac{\epsilon^{2}-1}{h^{2}}$ (as defined earlier in (30)).

Again we discuss our work of constructing the orbit. As we are considering up to the first order correction terms of $Q^{2}$, we can drop the $Q^{2} q^{2}$ term from (52) in our approximation. The solution of (52) can be written as

$u=A_{q}+\frac{B_{q}}{\cosh ^{2}\left(\omega_{q} \phi\right)}+Q^{2} \frac{k_{q}}{\cosh ^{4}\left(\omega_{q} \phi\right)}$

where $A_{q}, B_{q}, k_{q}, \omega_{q}$ all are constant and their approximate expressions are once again provided in Appendix B.

The trajectory equation for the charged probe is

$$
\begin{aligned}
& \left(\frac{\mathrm{d} u}{\mathrm{~d} \phi}\right)^{2}=-Q^{2} u^{4}+2 M u^{3}-u^{2}\left(1+\frac{Q^{2}}{h^{2}}-\frac{Q^{2} q^{2}}{h^{2}}\right) \\
& +\frac{2}{h^{2}}\left(M-\frac{q Q E}{m}\right) u+\frac{\epsilon^{2}-1}{h^{2}} \equiv f(u) .
\end{aligned}
$$

This is the other principal result of our paper.

We now concentrate on circular trajectories. For the occurrence of circular orbits the conditions are as follows:

$$
\begin{aligned}
& f(u)=-Q^{2} u^{4}+2 M u^{3}-u^{2}\left(1+\frac{Q^{2}}{h^{2}}-\frac{Q^{2} q^{2}}{h^{2}}\right) \\
& +\frac{2}{h^{2}}\left(M-\frac{q Q E}{m}\right) u+\frac{\epsilon^{2}-1}{h^{2}}=0, \\
& f^{\prime}(u)=-4 Q^{2} u^{3}+6 M u^{2}-2 u\left(1+\frac{Q^{2}}{h^{2}}-\frac{Q^{2} q^{2}}{h^{2}}\right) \\
& +\frac{2}{h^{2}}\left(M-\frac{q Q E}{m}\right)=0 .
\end{aligned}
$$

We can easily obtain the expressions for the energy and angular momentum of a circular orbit of radius $r_{c}=\frac{1}{u_{c}}$ from the above two equations. The expressions for the energy is 


$$
\begin{aligned}
\epsilon^{2} & =\frac{E^{2}}{m^{2}} \\
& =\frac{\left(1-2 M u_{c}+Q^{2} u_{c}^{2}\right)^{2}+q Q u_{c}\left[\frac{E}{m}\left(1-4 M u_{c}+3 Q^{2} u_{c}^{2}\right)+q Q u_{c}^{2}\left(M-Q^{2} u_{c}\right)\right]}{1-3 M u_{c}+2 Q^{2} u_{c}^{2}} .
\end{aligned}
$$

Now, up to order $q Q$ (ignoring the term $q^{2} Q^{2}$ ), the expression can be written as

$$
\begin{aligned}
\epsilon^{2}= & \frac{\left(1-2 M u_{c}+Q^{2} u_{c}^{2}\right)^{2}}{\left(1-3 M u_{c}+2 Q^{2} u_{c}^{2}\right)} \\
& +q Q u_{c}\left[\frac{\left(1-4 M u_{c}+3 Q^{2} u_{c}^{2}\right)\left(1-2 M u_{c}+Q^{2} u_{c}^{2}\right)}{\left(1-3 M u_{c}+2 Q^{2} u_{c}^{2}\right)^{\frac{3}{2}}}\right] .
\end{aligned}
$$

Apart from some minor mismatch in numerical factors this result essentially agrees with $[1,2]$. Similarly for angular momentum, we have

$h^{2}=\frac{\left(M-Q^{2} u_{c}\right)-q Q\left(\frac{E}{m}-q Q u_{c}\right)}{u_{c}\left(1-3 M u_{c}+2 Q^{2} u_{c}^{2}\right)}$.

Thus, up to order of $q Q$,

$$
\begin{aligned}
h^{2}= & \frac{\left(M-Q^{2} u_{c}\right)}{u_{c}\left(1-3 M u_{c}+2 Q^{2} u_{c}^{2}\right)} \\
& -q Q\left[\frac{\left(1-2 M u_{c}+Q^{2} u_{c}^{2}\right)}{u_{c}\left(1-3 M u_{c}+2 Q^{2} u_{c}^{2}\right)^{\frac{3}{2}}}\right] .
\end{aligned}
$$

There is an interesting observation regarding a possible scaling of the charges following [19] where variations of $\epsilon^{2}=(E / m)^{2}$ and $h=l / m$ against $M u_{c}$ are discussed with the scaling $Q^{2}=p M^{2}, p$ being a numerical constant. The resulting relation for the latter for a neutral probe is [19]

$\frac{h^{2}}{M^{2}}=\frac{\left(1-p M u_{c}\right)}{M u_{c}\left(1-3 M u_{c}+2 p M^{2} u_{c}^{2}\right)}$.

However, if we consider an identical scaling in our present case with a charged probe, the relation turns out to be

$$
\begin{aligned}
\frac{h^{2}}{M^{2}}= & \frac{\left(1-p M u_{c}\right)}{M u_{c}\left(1-3 M u_{c}+2 p M^{2} u_{c}^{2}\right)} \\
& -q \sqrt{p}\left[\frac{\left(1-2 M u_{c}+p M^{2} u_{c}^{2}\right)}{M u_{c}\left(1-3 M u_{c}+2 p M^{2} u_{c}^{2}\right)^{\frac{3}{2}}}\right] .
\end{aligned}
$$

The appearance of the parameter $q$ is indicative of the fact that the Coulomb force is essentially non-geometric and hence the trajectories are not pure geodesic in nature.

The effect of the probe charge, especially whether it is of the same sign as the black hole charge or of opposite sign, is quite striking. Intuitively we can argue that for the opposite sign case the probe charge effect will not be very significant

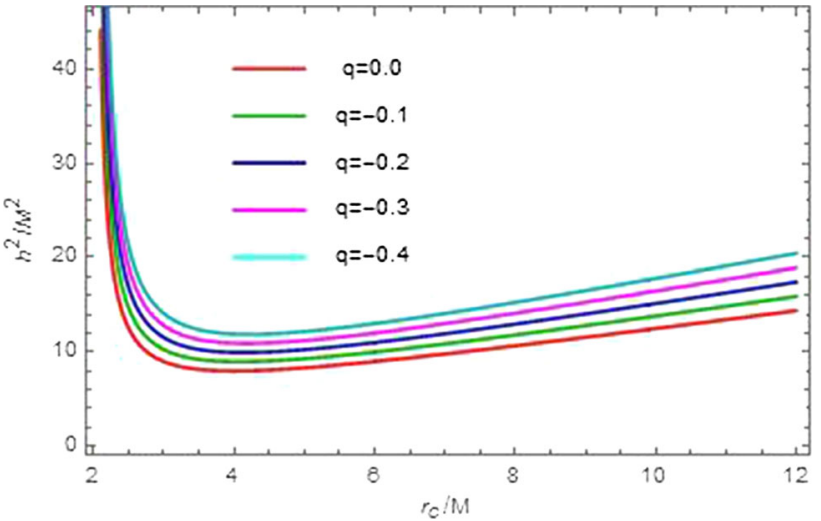

Fig. $1 h^{2} / M^{2}$ vs. $r_{c} / M$ are plotted for fixed $Q^{2}=p M^{2}, p=1$ and different negative values of $q$. The curves of charged probes are always above the neutral probe but of same qualitative nature

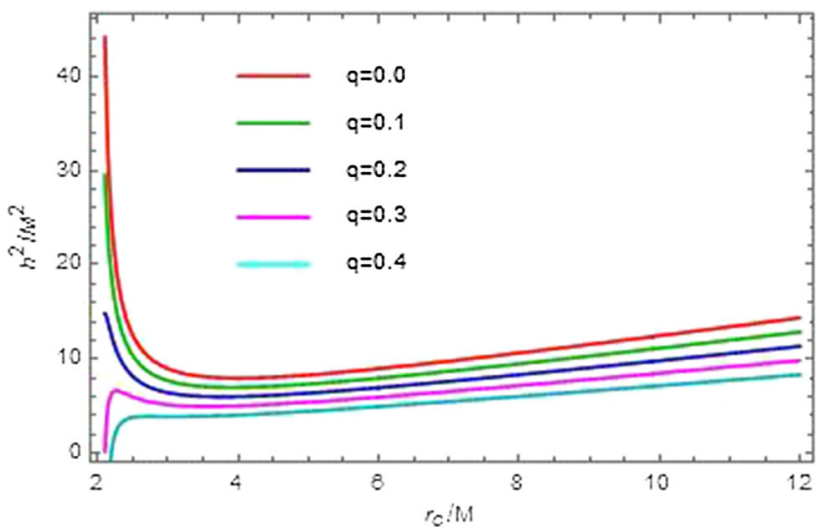

Fig. $2 h^{2} / M^{2}$ vs. $r_{c} / M$ are plotted for fixed $Q^{2}=p M^{2}, p=1$ and different positive values of $q$. The curves of charged probes are always below the neutral probe. The nature of the curve changes for $q>0.2$ and becomes unphysical at $\sim 0.3$ onwards

because both the gravitational force and the Coulomb force will be attractive and so qualitatively similar behavior to the neutral case will be observed. This is shown in Fig. 1.

On the other hand, if the probe and black hole charges are of the same sign the Coulomb force will be repulsive whereas the gravitational force is attractive as before. The interplay between these two forces produces an upper bound of the $q$ parameter above which the results become unphysical. This is demonstrated in Fig. 2.

A similar behavior for $\epsilon^{2}=(E / m)^{2}$ vs. $r_{c} / M$ is observed in Figs. 3 and 4 where negative and positive values of $q$ are considered, respectively.

The minimum radius for a stable circular orbit will occur at the point of inflection of the function $f(u)$, i.e.,

$$
f^{\prime \prime}(u)=-12 Q^{2} u^{2}+12 M u-2\left(1+\frac{Q^{2}}{h^{2}}-\frac{Q^{2} q^{2}}{h^{2}}\right)
$$




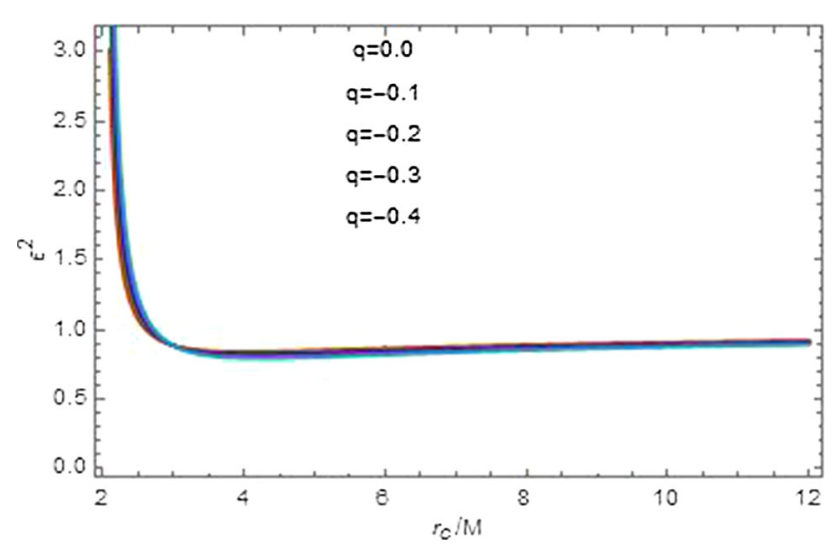

Fig. $3 \epsilon^{2}$ vs. $r_{c} / M$ are plotted for fixed $Q^{2}=p M^{2}, p=1$ and different negative values of $q$

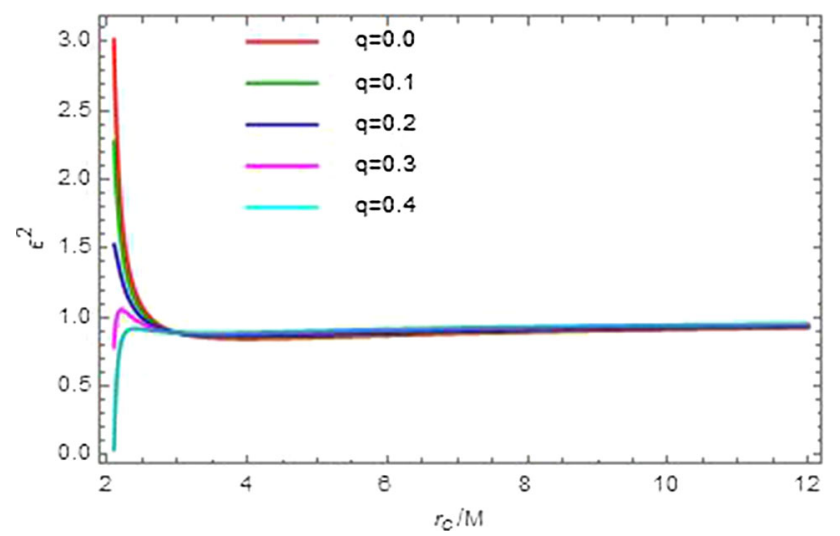

Fig. $4 \epsilon^{2}$ vs. $r_{c} / M$ are plotted for fixed $Q^{2}=p M^{2}, p=1$ and different positive values of $q$

Eliminating $h^{2}$ from the above equation using (59), we can write

$$
\begin{aligned}
& 4 Q^{4} u_{c}^{3}-9 M Q^{2} u_{c}^{2}+6 M^{2} u_{c}-M-q Q \\
& {\left[q Q\left(4 u_{c}^{3} Q^{2}-3 M u_{c}^{2}\right)-\frac{E}{m}\left(6 Q^{2} u_{c}^{2}-6 M u_{c}+1\right)\right]=0}
\end{aligned}
$$

or, in terms of $r_{c}$,

$$
\begin{aligned}
& r_{c}^{3}-6 M r_{c}^{2}+9 Q^{2} r_{c}-\frac{4 Q^{4}}{M} \\
& -\frac{q Q E}{m}\left(\frac{r_{c}^{3}}{M}-6 r_{c}^{2}+\frac{6 r_{c} Q^{2}}{M}\right) \\
& -q^{2} Q^{2}\left(3 r_{c}-\frac{4 Q^{2}}{M}\right)=0 .
\end{aligned}
$$

Let us define a parameter $\Lambda=(q Q E) /(m M)$, in terms of which the above equation is rewritten as

$$
\begin{aligned}
& r_{c}^{3}(1-\Lambda)-6 M r_{c}^{2}(1-\Lambda) \\
& \quad+9 Q^{2} r_{c}\left(1-\frac{2}{3} \Lambda-\frac{q^{2}}{3}\right)-\frac{4 Q^{4}}{M}\left(1-q^{2}\right)=0 .
\end{aligned}
$$

Ignoring $O\left(Q^{4}\right)$ terms we obtain

$r_{c}=3 M \pm 3 M \sqrt{1-\frac{Q^{2}\left(1-\frac{2}{3} \Lambda-\frac{q^{2}}{3}\right)}{M^{2}(1-\Lambda)}}$.

Note that, for $q=0$, that is, a neutral probe, the earlier result (48),

$$
r_{c}=6 M-\frac{3 Q^{2}}{2 M}+\cdots
$$

is recovered. Considering $q$ and $\Lambda$ to be small (which is quite natural) we find

$r_{c}=6 M-\frac{3 Q^{2}}{2 M}\left(1+\frac{\Lambda}{3}\right)$.

This shows that the effective charge of the black hole increases for positive $\Lambda$, that is, when the probe charge $q$ and black hole charge $Q$ are of the same sign but it decreases when they are of opposite sign. This concludes our discussion of the charged particle trajectory in the presence of a charged black hole.

\section{Gaussian curvature for Jacobi metric}

A study of the Gaussian curvature for the Jacobi metric for the Reissner-Nordström black hole, as experienced by a particle of fixed energy $E$, is the other major contribution of our work. The Jacobi metric corresponding to the Reissner-Nordström geometry (20) (considering the system in the equatorial plane $\left.\theta=\frac{\pi}{2}\right)$

$$
\begin{aligned}
\mathrm{d} s^{2}= & \left(E^{2}-m^{2}+\frac{2 M m^{2}}{r}-\frac{Q^{2} m^{2}}{r^{2}}\right) \\
& \times\left[\frac{\mathrm{d}^{2}}{\left(1-\frac{2 M}{r}+\frac{Q^{2}}{r^{2}}\right)^{2}}+\frac{r^{2} \mathrm{~d} \phi^{2}}{\left(1-\frac{2 M}{r}+\frac{Q^{2}}{r^{2}}\right)}\right]
\end{aligned}
$$

vanishes when

$$
E^{2}=m^{2}\left(1-\frac{2 M}{r}+\frac{Q^{2}}{r^{2}}\right) .
$$


The equation saturates for a critical value $r_{c}$ :

$$
\begin{aligned}
r_{c} & =\frac{M m^{2}\left(1+\left(1-\frac{\left(m^{2}-E^{2}\right) Q^{2}}{M^{2} m^{2}}\right)^{\frac{1}{2}}\right)}{m^{2}-E^{2}} \\
& \approx 2 M\left(\frac{m^{2}}{m^{2}-E^{2}}-\frac{Q^{2}}{4 M^{2}}\right)+O\left(Q^{4}\right) .
\end{aligned}
$$

This leads to an inequality of the form $\frac{m^{2}}{m^{2}-E^{2}} \geq \frac{Q^{2}}{4 M^{2}}$ and since $\frac{m^{2}}{m^{2}-E^{2}}<1$, one has $Q<2 M$. It is interesting to recall that $Q=M$ is the extremality condition and, moreover, $Q<M$ is generally assumed to avoid the presence of a naked singularity (or violation of the cosmic censorship hypothesis by Penrose). Hence $Q<2 M$ derived above is a weaker condition and does not add any further restrictions on the charge-mass ratio.

Interpreting the following expression as a Jacobi circumference [8]:

$2 \pi\left(E^{2}-m^{2}+\frac{2 M m^{2}}{r}-\frac{Q^{2} m^{2}}{r^{2}}\right)^{\frac{1}{2}} \frac{r}{\left(1-\frac{2 M}{r}+\frac{Q^{2}}{r^{2}}\right)^{\frac{1}{2}}}$

the boundary $r_{c}$ actually reduces to a point since the circumference vanishes there.

A condition for circular geodesics is derived from the extrema of the Jacobi circumference,

$\frac{E^{2}}{m^{2}}=\frac{\left(1-\frac{2 M}{r}+\frac{Q^{2}}{r^{2}}\right)^{2}}{\left(1-\frac{3 M}{r}+\frac{2 Q^{2}}{r^{2}}\right)}$.

Circular orbits exist provided $\left(1-\frac{3 M}{r}+\frac{2 Q^{2}}{r^{2}}\right) \geq 0$. This means that to $O\left(Q^{2} / M\right)$ the roots are

$r_{+}=3 M-\left(2 Q^{2}\right) /(3 M), \quad r_{-}=\left(2 Q^{2}\right) /(3 M)$,

indicating that $r \geq 3 M-\left(2 Q^{2}\right) /(3 M)$.

Several examples of radii (to $O\left(Q^{2}\right)$ ) for some specific values of energy are provided below: for $m^{2}=0$, the term $\left(1-\frac{3 M}{r}+\frac{2 Q^{2}}{r^{2}}\right)$ must be equal to zero, leading to $r \approx 3 M-\frac{2 Q^{2}}{3 M}$. The outermost and innermost circular orbit radii are computed by extremizing the r.h.s. of (72). These are, respectively, $r \approx 6 M-\frac{3 Q^{2}}{2 M}$ for $E^{2} \approx m^{2}\left[\frac{8}{9}-\frac{2 Q^{2}}{81 M^{2}}\right]$ and $r \approx 4 M-\frac{Q^{2}}{M}$ for $E^{2}=m^{2}$. Incidentally the last example agrees with our previously derived result (48). For $Q=0$ the above reduce to the Schwarzschild geometry results [8].

Let us now come to the explicit structure of the Gaussian curvature $K$. As a warm-up exercise let us compute $K_{S}$ for the Schwarzschild case. Expressing the Jacobi metric pertaining to the Schwarzschild geometry as

$\mathrm{d} s^{2}=f(r)^{2}\left[\mathrm{~d} r^{2}+r^{2}\left(1-\frac{2 M}{r}\right) \mathrm{d} \phi^{2}\right]$

where

$f(r)^{2}=\left(E^{2}-m^{2}+\frac{2 M m^{2}}{r}\right) \frac{1}{\left(1-\frac{2 M}{r}\right)^{2}}$

the Gaussian curvature $K_{S}$ is given by

$$
\begin{aligned}
K_{S} & =-\frac{1}{f(r)^{2} r\left(1-\frac{2 M}{r}\right)^{\frac{1}{2}}} \frac{\mathrm{d}}{\mathrm{d} r}\left[\frac{1}{f(r)} \frac{\mathrm{d}}{\mathrm{d} r}\left(r f(r)\left(1-\frac{2 M}{r}\right)^{\frac{1}{2}}\right)\right] \\
& =\frac{M\left[m^{4}(2 M-r)^{3}+E^{4} r^{2}(3 M-2 r)+3 E^{2} m^{2} r(2 M-r)(M-r)\right]}{r^{3}\left[m^{2}(2 M-r)+E^{2} r\right]^{3}} .
\end{aligned}
$$

For a massless probe $m=0$, the curvature simplifies to

$K_{S}=-\frac{2 M}{E^{2} r^{3}}\left(1-\frac{3 M}{2 r}\right)$.

For $r \geq 2 M, K_{S}$ is always negative $[14,15]$.

\section{Uncharged probe}

Let us start with a massless neutral probe. In a similar way to above, for the Reissner-Nordström case, the Gaussian curvature to $O\left(Q^{2}\right)$ is given by

$K_{R N}=K_{S}+(A / B) Q^{2}$

where

$$
\begin{aligned}
A= & 3 E^{6} r^{3}(-2 M+r)-m^{6}(-2 M+r)^{4} \\
& +E^{4} m^{2} r^{2}\left(-16 M^{2}+22 M r-7 r^{2}\right) \\
& +E^{2} m^{4} r\left(-28 M^{3}+42 M^{2} r-24 M r^{2}+5 r^{3}\right), \\
B= & r^{4}\left[m^{2}(2 M-r)+E^{2} r\right]^{4} .
\end{aligned}
$$

For $m=0$, a massless probe, the Gaussian curvature reduces to the form

$K_{R N}=-\frac{1}{E^{2}}\left[\frac{2 M}{r^{3}}\left(1-\frac{3 M}{2 r}\right)-\frac{3 Q^{2}}{r^{4}}\left(1-\frac{2 M}{r}\right)\right]$.

Comparing with the $Q=0$ case (76), the situation becomes more complicated and indeed, it is possible that the $Q$-contribution might reverse the sign of $K_{R N}$. To verify this we define a convenient scaling $E^{2}=c_{1} M^{2}, r=a M, Q=$ $b M$ and rewrite $K_{R N}$ in (79) as

$K_{R N}=-\frac{1}{a^{3} c_{1} M^{4}}\left[2\left(1-\frac{3}{2 a}\right)-\frac{3 b^{2}}{a}\left(1-\frac{2}{a}\right)\right]$.

To see the effect of $Q$ we plot $K_{R N}$ vs. $c_{1}=E^{2} / M^{2}$ for fixed values of $b$ in Fig. 5, near the outer boundary where $a=6-\frac{3}{2} b^{2}$. 


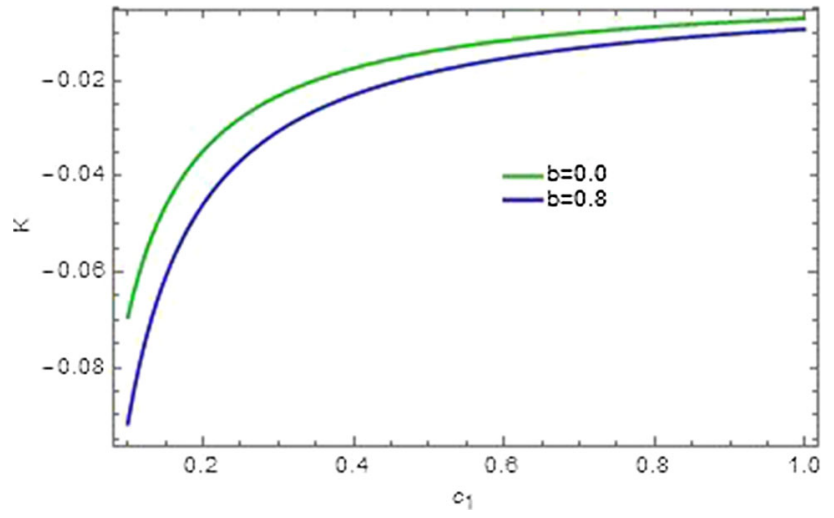

Fig. $5 K_{R N}$ vs. $c_{1}=E^{2} / M^{2}$ are plotted for $Q=b M$ where $b$ takes different positive values near the outer boundary

The graphs indicate that, to the order of approximation we are considering, for a massless probe, $K_{R N}$ stays negative, as in the Schwarzschild case [8] and, as the energy of the particle increases, the magnitude of the Gaussian curvature decreases and asymptotically tends to zero. Thus for a massless probe, the presence of a black hole charge has not too much effect except that it increases the negativity of the Gaussian curvature.

Let us now consider a probe with mass $m$ where the dynamics changes qualitatively from the massless probe discussed above. We introduce the parameterization $E^{2}=$ $\mathrm{cm}^{2}, r=a M, Q=b M$ and plot $K_{R N}$ vs. $c=E^{2} / \mathrm{m}^{2}$ (Figs. 6, 7, and 8). The Gaussian curvature (to order $Q^{2}$ ), near the outer boundary $a=6-\frac{3}{2} b^{2}$, is

$$
\begin{aligned}
K_{R N}= & \frac{-64+360 c-324 c^{2}}{216(-4+6 c)^{3} m^{2} M^{2}} \\
& +\frac{\left(28-201 c+333 c^{2}-162 c^{3}\right) b^{2}}{648(-2+3 c)^{4} m^{2} M^{2}} .
\end{aligned}
$$

From Fig. 6 we notice that, for the energy range $0.7<$ $E^{2} / m^{2}<0.8$, for small $b^{2}$ (small $Q$ ), the Gaussian curvature $K_{R N}$ is positive but it changes sign and becomes negative for larger $Q \sim 0.5$ and from Fig. 7 we see that the transition occurs around $b^{2}=0.3$. Interestingly in this case the $K_{R N}$ starts with a negative value and briefly reaches positive values before vanishing asymptotically. From Fig. 8, we find that, for $c=E^{2} / m^{2} \leq 0.2, K_{R N}$ remains positive both for $Q=0$ and for non-zero $Q$.

For the inner boundary, we put $a=4-b^{2}$ and $K_{R N}$ is

$K_{R N}=\frac{-8+72 c-80 c^{2}}{64(-2+4 c)^{3} m^{2} M^{2}}+\frac{\left(2-23 c+44 c^{2}-24 c^{3}\right) b^{2}}{256(-1+2 c)^{4} m^{2} M^{2}}$.

We have chosen the same value $b^{2}=0.3$ for which the sign change occurred at outer boundary. The plot in Fig. 9 indicates that, near the inner boundary, for $b^{2}=0.3, K_{R N}$

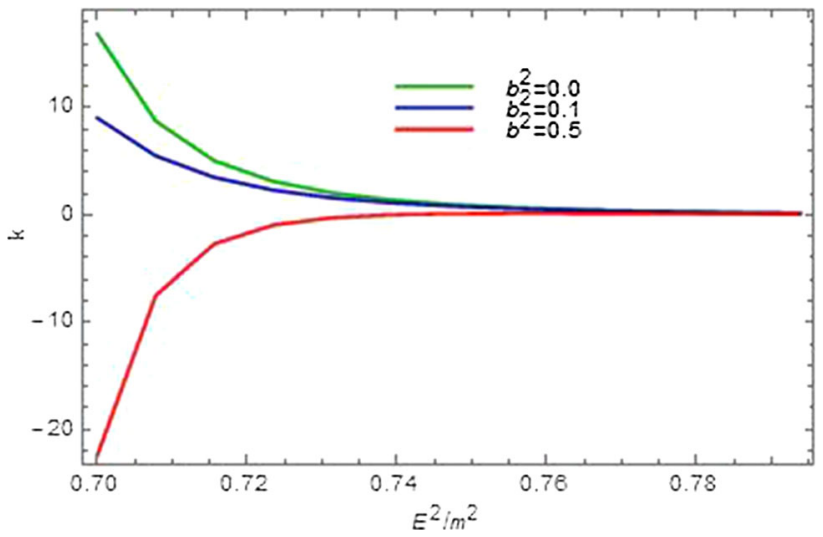

Fig. $6 K_{R N}$ vs. $c=E^{2} / m^{2}$ are plotted for $Q^{2}=b^{2} M^{2}$ and different positive values of $b^{2}$ near the outer boundary $\left(0.7<E^{2} / m^{2}<0.8\right)$

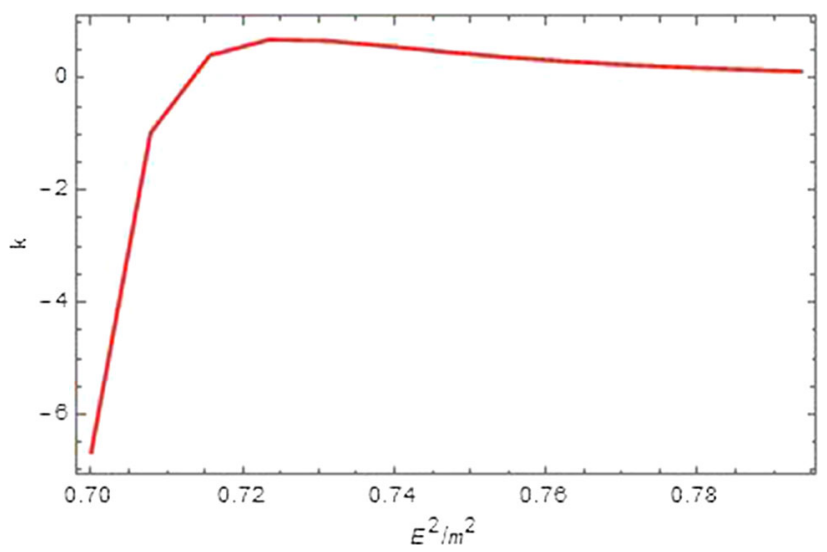

Fig. $7 K_{R N}$ vs. $c=E^{2} / m^{2}$ are plotted for $Q^{2}=b^{2} M^{2}$ where $b^{2}=0.3$ near the outer boundary $\left(0.7<E^{2} / m^{2}<0.8\right)$

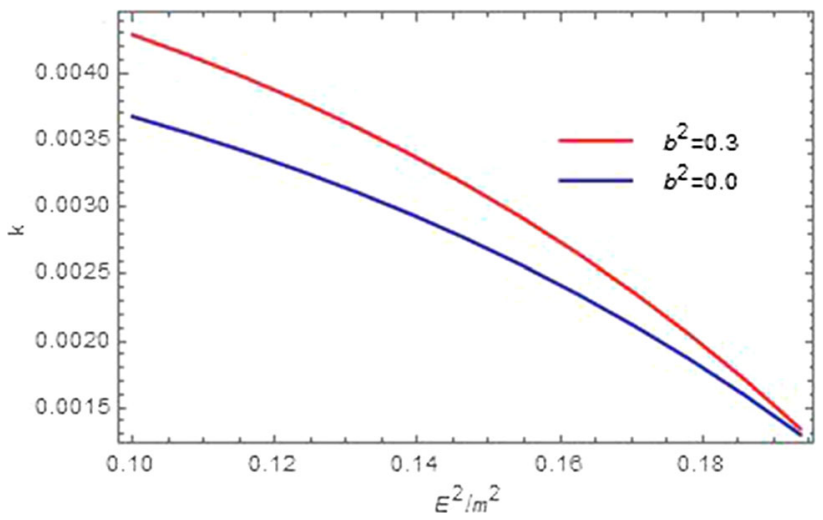

Fig. $8 K_{R N}$ vs. $c=E^{2} / m^{2}$ are plotted for $Q^{2}=b^{2} M^{2}$ and different positive values of $b^{2}$ near the outer boundary $\left(E^{2} / m^{2}<0.2\right)$

tends to become more negative but the graphs shows a similar qualitative nature for both zero and non-zero $Q$.

\section{Charged probe}

We now discuss the case of charged probe where an additional Coulomb interaction needs to be taken into account. 


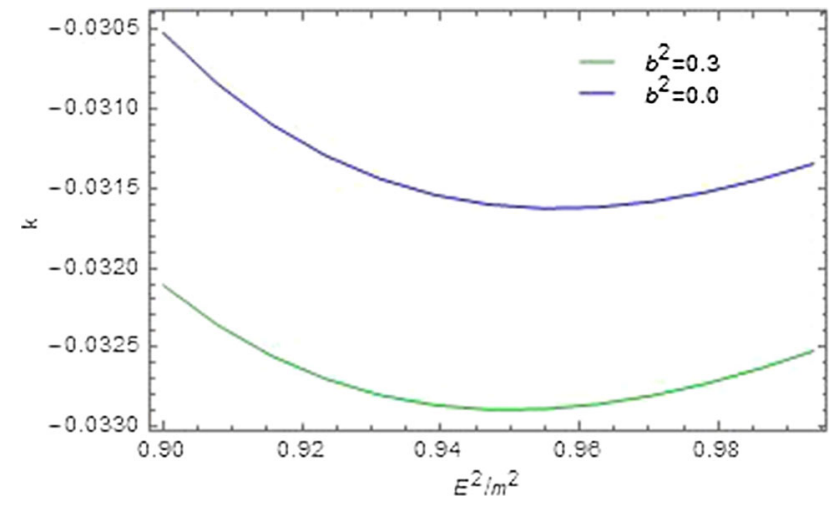

Fig. $9 K_{R N}$ vs. $c=E^{2} / m^{2}$ are plotted for $Q^{2}=b^{2} M^{2}$ and different positive values of $b^{2}$ near the inner boundary $\left(0.9<E^{2} / m^{2}<1\right)$

The Jacobi metric corresponding to the Reissner-Nordström case with a charged probe $(q)$ (in the equatorial plane) is

$$
\begin{aligned}
\mathrm{d} s^{2}= & \left(\left(E-\frac{m q Q}{r}\right)^{2}-m^{2}+\frac{2 M m^{2}}{r}-\frac{Q^{2} m^{2}}{r^{2}}\right) \\
& \times\left[\frac{\mathrm{d} r^{2}}{\left(1-\frac{2 M}{r}+\frac{Q^{2}}{r^{2}}\right)^{2}}+\frac{r^{2} \mathrm{~d} \phi^{2}}{\left(1-\frac{2 M}{r}+\frac{Q^{2}}{r^{2}}\right)}\right] .
\end{aligned}
$$

The metric vanishes when

$$
E^{2}-\frac{2 E m Q q}{r}=m^{2}\left(1-\frac{2 M}{r}+\frac{Q^{2}}{r^{2}}\right)
$$

where the $q^{2} Q^{2}$-term has been dropped for small $q$. This leads to

$r_{c}=\frac{\left(M m^{2}-E m Q q\right)\left(1+\left(1-\frac{\left(m^{2}-E^{2}\right) Q^{2}}{(M m-E Q q)^{2}}\right)^{\frac{1}{2}}\right)}{m^{2}-E^{2}}$.

Again this can be thought of as a point where the Jacobi circumference vanishes. The circumference can be written from the metric as

$$
\begin{aligned}
2 \pi & \left(E^{2}-m^{2}-\frac{2 E m Q q}{r}+\frac{2 M m^{2}}{r}-\frac{Q^{2} m^{2}}{r^{2}}\right)^{\frac{1}{2}} \\
& \times \frac{r}{\left(1-\frac{2 M}{r}+\frac{Q^{2}}{r^{2}}\right)^{\frac{1}{2}}} .
\end{aligned}
$$

Circular geodesics correspond to the extrema of the Jacobi circumference for which

$$
\begin{aligned}
\frac{E^{2}}{m^{2}}= & \frac{\left(1-\frac{2 M}{r}+\frac{Q^{2}}{r^{2}}\right)^{2}}{\left(1-\frac{3 M}{r}+\frac{2 Q^{2}}{r^{2}}\right)} \\
& \pm \frac{Q q}{r}\left[\frac{\left(1-\frac{4 M}{r}+\frac{3 Q^{2}}{r^{2}}\right)\left(1-\frac{2 M}{r}+\frac{Q^{2}}{r^{2}}\right)}{\left(1-\frac{3 M}{r}+\frac{2 Q^{2}}{r^{2}}\right)^{\frac{3}{2}}}\right] .
\end{aligned}
$$

For the Reissner-Nordström geometry where the probe charge $q$ is present, the Gaussian curvature can be written as

$K_{R N P}=K_{S}+(C / D) q Q$

where

$$
\begin{aligned}
C= & E m\left[E^{4} r^{2}\left(12 M^{2}-9 M r+r^{2}\right)\right. \\
& \left.+m^{4}(-2 M+r)^{2}\left(4 M^{2}-M r+r^{2}\right)\right] \\
& -2 E^{3} m^{3} r\left(-4 M^{3}+12 M^{2} r-7 M r^{2}+r^{3}\right), \\
D= & r^{3}\left[m^{2}(2 M-r)+E^{2} r\right]^{4} .
\end{aligned}
$$

It is worthwhile to point out that the leading correction term depends on $q Q$, both linearly on $q$ and on $Q$, which leads to interesting consequences. Firstly, the relative sign of black hole charge $Q$ and probe charge $q$ becomes important as it dictates the nature of Coulomb interaction, that is, whether it is repulsive or attractive. Secondly, unlike the ReissnerNordström geometry with a neutral probe, here we can drop $Q^{2}$-terms due to the presence of $q Q$-terms.

Now where the probe charge $q$ is present, we parametrize $Q=M / 2, r=\frac{15}{8} p M$ and $E^{2} / m^{2}=1$ and find the Gaussian curvature,

$$
K=-\frac{8(-32+45 p)(2+q)}{3375 M^{2} p^{3}} .
$$

From the above expression we can easily observe that, for positive values of the probe charge $q$ or in the absence of the probe charge, the graphs will show a similar qualitative nature (shown in Fig. 10) whereas, for negative values of the probe charge, i.e. for $(2+q)<0$ or $q<-2$, it will reverse the sign of the Gaussian curvature $K$ and the curvature starts with negative value and tends to zero.

Similarly, if we plot $K$ vs. $c=E^{2} / m^{2}$ for negative and positive values of probe charge $q$, the graphs show a similar qualitative nature for positive values of the probe charge whereas it shows a reverse nature for negative values of the probe charge (shown in Fig. 11). 


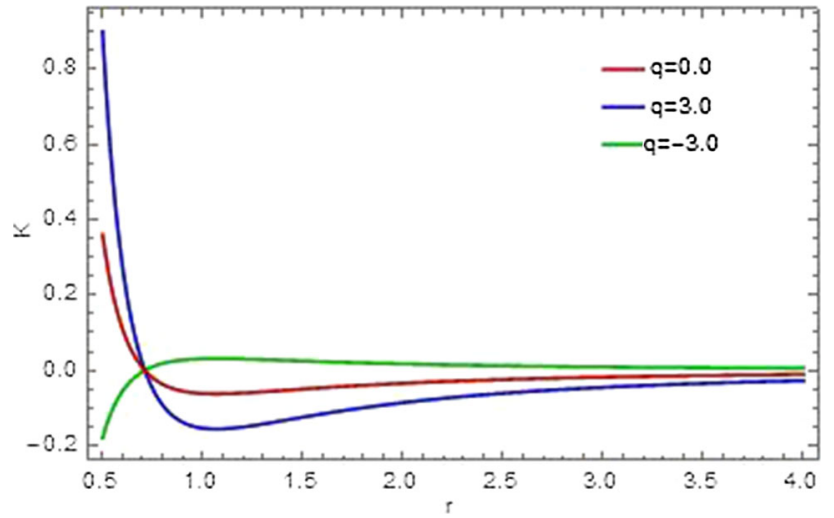

Fig. $10 K$ vs. $r$ are plotted for positive and negative values of the probe charge $q$ for fixed $Q=M / 2$

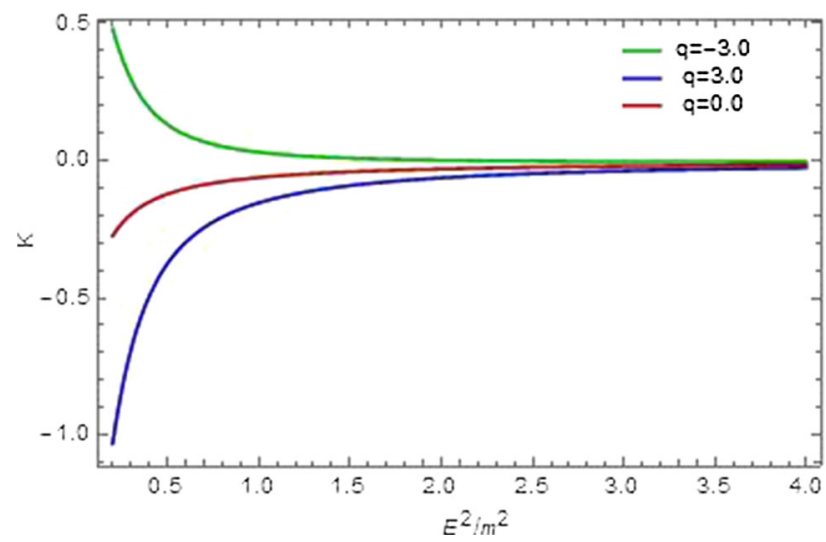

Fig. $11 K$ vs. $c=E^{2} / m^{2}$ are plotted for positive and negative values of the probe charge $q$ for fixed $Q=M / 2$

\section{Conclusion}

In the present work we have considered particle trajectories that are parameterized by constant energy value. This feature helps to visualize quickly the bounded and unbounded nature of particle orbits related to particle energy. This characteristic is very succinctly incorporated in the Jacobi extension of least action principle. The formalism starts with the construction of the Jacobi metric where the (conserved) particle energy appears explicitly in the metric. As has been proved by Pin [13] a restricted variational principle à la Maupertuis with conventional metric and constant particle energy is equivalent to an unrestricted variational principle with Jacobi metric, which explicitly involves the particle energy. Hence the constant energy paths are still geodesics but of the Jacobi metric.

Exploiting this formalism we have studied the worldlines of both uncharged and charged probes in a ReissnerNordström background. The former is a straightforward generalization of the Schwarzschild black hole as given by Gibbons [8] whereas the latter is a non-trivial extension, since it has an additional Coulomb interaction. In both cases we have derived the circular orbit condition. The relative sign of the probe and black hole (whether the two have the same sign or opposite sign) plays a significant role in determining the nature of the particle trajectory. Indeed, it should be stressed that there already exist series of recent [1] and earlier publications [2,3] by Pugliese et al. where this problem has been treated in detail in an exact way from a graphical perspective. We, on the other hand, have attempted to complement their work by providing analytic form of the orbits in an approximate scenario of small charge for the black hole and probe. We have also given expressions for the generic closed orbit and its reduction to circular orbits.

The Gauss curvature pertaining to Jacobi metric comprises another new and interesting aspect of our work. In earlier work $[8,13]$, the Gaussian curvature of the Jacobi metric played an important role in characterizing the nature of the particle worldlines in terms of open or closed orbits related to the particle energy. We have studied the properties of Gaussian curvature related to Jacobi metric for uncharged probe (massless and massive case) and also for the charged probe.

For massless uncharged probe, the presence of black hole charge $Q$ only increases the negativity of Gaussian curvature, whereas for the massive case it plays a significant role i.e. the black hole charge can reverse the sign of Gaussian curvature for holes with charge-mass ratio above a certain value.

When the probe is charged, an additional Coulomb interaction comes into play and the sign of the probe charge, whether it is of same sign or opposite to the black hole charge, becomes crucial. It is expected that if the charges are of the same sign, the effect becomes pronounced due to the competition between the repulsive Coulomb force and attractive gravitational force. On the other hand, the effect will be weaker if the charges are of opposite sign since both the Coulomb and the gravitational forces will be attractive in nature. This is manifested in the angular momentum vs. circular radius and energy vs. circular radius graphs. However, curiously enough, the Gaussian curvature behaves in a different way. For a positively charged black hole, if the probe charge is also positive, the Gaussian curvature starts with a negative value and asymptotically tends to zero. This is similar to the case of a neutral probe. But if the probe has a negative charge $(q<-2)$, (opposite to the positive black hole charge), the curvature stays positive throughout and asymptotes to zero.

Acknowledgements It is a pleasure to thank Professor Gary Gibbons for much helpful correspondence and for sending us reprints of early references. We are grateful to the referee for helpful comments that have helped to improve presentation of the paper. P.D. acknowledges the financial support from INSPIRE, DST, India.

Open Access This article is distributed under the terms of the Creative Commons Attribution 4.0 International License (http://creativecomm 
ons.org/licenses/by/4.0/), which permits unrestricted use, distribution, and reproduction in any medium, provided you give appropriate credit to the original author(s) and the source, provide a link to the Creative Commons license, and indicate if changes were made.

Funded by SCOAP ${ }^{3}$.

\section{Appendix A}

Explicit solutions for the constants given in [8] for Schwarzschild background are

$$
\begin{aligned}
A_{G} & =\frac{1}{6 M}\left(1 \pm \sqrt{1-\frac{12 M^{2}}{h^{2}}}\right)=\frac{1}{6 M}\left(1-2 M B_{G}\right) \\
B_{G} & =\mp \frac{1}{2 M} \sqrt{1-\frac{12 M^{2}}{h^{2}}} \\
\omega_{G}^{2} & = \pm \frac{1}{4} \sqrt{1-\frac{12 M^{2}}{h^{2}}}=-\frac{M B_{G}}{2} \\
C_{G} & =A_{G}^{2}\left(4 M A_{G}-1\right) \\
& =\frac{1}{36 M^{2}}\left(1-2 M B_{G}\right)^{2}\left[-\frac{4}{3} M B_{G}-\frac{1}{3}\right] .
\end{aligned}
$$

Exploiting these we provide below the $O\left(Q^{2}\right)$ corrected expressions for the neutral probe Reissner-Nordström system,

$$
\begin{aligned}
& A=A_{G}+Q^{2} f, \quad B=B_{G}+Q^{2} g, \quad \omega^{2}=\omega_{G}^{2}+Q^{2} s, \\
& C=C_{G}+Q^{2} t
\end{aligned}
$$

where

$$
\begin{aligned}
f & =\frac{2 A_{G}^{3}+\frac{A_{G}}{h^{2}}}{6 M A_{G}-1} \\
& =-\frac{\left(1-2 M B_{G}\right)}{2 M B_{G}}\left[\frac{1}{108 M^{3}}\left(1-2 M B_{G}\right)^{2}+\frac{1}{6 M h^{2}}\right], \\
g & =\frac{-2 B_{G} s+4 k \omega_{G}^{2}+2 A_{G} B_{G}^{2}}{M B_{G}} \\
& =\frac{1}{M B_{G}}\left[-2 B_{G} s \pm B_{G}^{3}+\frac{1}{3 M}\left(1-2 M B_{G}\right) B_{G}^{2}\right] \\
s & =\frac{1}{4}\left[-6 A_{G}^{2} \mp 3 A_{G}^{2}+6 M f \pm \frac{A_{G}}{M}-\frac{1}{h^{2}} \mp \frac{1}{h^{2}}\right]
\end{aligned}
$$

or equivalently

$$
\begin{aligned}
s= & \frac{1}{4}\left[\left(-\frac{1}{6 M^{2}} \mp \frac{1}{12 M^{2}}\right)\left(1-2 M B_{G}\right)^{2}\right. \\
& \left.+6 M f \pm \frac{1}{6 M^{2}}\left(1-2 M B_{G}\right)-\frac{1}{h^{2}} \mp \frac{1}{h^{2}}\right], \\
t= & A_{G}^{2}\left(A_{G}^{2}+\frac{1}{h^{2}}\right)
\end{aligned}
$$

$$
\begin{aligned}
& =\frac{1}{36 M^{2}}\left(1-2 M B_{G}\right)^{2}\left[\frac{1}{36 M^{2}}\left(1-2 M B_{G}\right)^{2}+\frac{1}{h^{2}}\right] \\
k & =\mp \frac{1}{8 M^{3}}\left(1-\frac{12 M^{2}}{h^{2}}\right)=\mp \frac{B_{G}^{2}}{2 M}
\end{aligned}
$$

\section{Appendix B}

Similarly, the $O\left(Q^{2}\right)$ corrected expressions for the charged probe Reissner-Nordström system are given by

$$
\begin{aligned}
& A_{q}=A_{G}+Q^{2} f, \quad B_{q}=B_{G}+Q^{2} g, \quad \omega_{q}^{2}=\omega_{G}^{2}+Q^{2} s, \\
& C_{q}=C_{G}+Q^{2} t,
\end{aligned}
$$

but the changes appeared only for the expressions of $A_{q}$ and $C_{q}$.

$$
\begin{aligned}
A_{q}= & A_{G}+Q^{2} \frac{\left(2 M B_{G}-1\right)}{2 M B_{G}}\left[\frac{1}{108 M^{3}}\left(1-2 M B_{G}\right)^{2}+\frac{1}{6 M h^{2}}\right] \\
& +Q q\left[\frac{E}{h^{2}\left(-2 M B_{G}\right)}\right], \\
B_{q}= & B_{G}+Q^{2} \frac{1}{M B_{G}}\left[-2 B_{G} s \pm B_{G}^{3}+\frac{1}{3 M}\left(1-2 M B_{G}\right) B_{G}^{2}\right],
\end{aligned}
$$

$$
\begin{aligned}
\omega_{q}^{2}= & \omega_{G}^{2}+\frac{Q^{2}}{4}\left[\left(-\frac{1}{6 M^{2}} \mp \frac{1}{12 M^{2}}\right)\left(1-2 M B_{G}\right)^{2}+6 M f\right. \\
& \left. \pm \frac{1}{6 M^{2}}\left(1-2 M B_{G}\right)-\frac{1}{h^{2}} \mp \frac{1}{h^{2}}\right] \\
C_{q}= & C_{G}+Q^{2} \frac{1}{36 M^{2}}\left(1-2 M B_{G}\right)^{2}\left[\frac{1}{36 M^{2}}\left(1-2 M B_{G}\right)^{2}+\frac{1}{h^{2}}\right] \\
& +Q q\left[\frac{E}{3 M h^{2}}\left(1-2 M B_{G}\right)\right], \\
k_{q}= & \mp \frac{B_{G}^{2}}{2 M}
\end{aligned}
$$

\section{References}

1. D. Pugliese, H. Quevedo, R. Ruffini, Eur. Phys. J. C 77, 206 (2017). arXiv: 1304.2940

2. D. Pugliese, H. Quevedo, R. Ruffini, Phys. Rev. D 88, 024042 (2013). arXiv:1303.6250

3. D. Pugliese, H. Quevedo, R. Ruffini, Phys. Rev. D 84, 044030 (2011). arXiv:1105.2959

4. D. Pugliese, H. Quevedo, R. Ruffini, Eur. Phys. J. C 71, 1638 (2011)

5. D. Pugliese, H. Quevedo, R. Ruffini, Phys. Rev. D 83, 104052 (2011)

6. D. Pugliese, H. Quevedo, R. Ruffini, Phys. Rev. D 83, 024021 (2011). arXiv:1012.5411

7. S. Grunau, V. Kagramanova, Phys. Rev. D 83, 044009 (2011). arXiv:1011.5399 [gr-qc]

8. G.W. Gibbons, Class. Quantum Grav. 33, 025004 (2016). arXiv: 1508.06755

9. S. Chanda, G.W. Gibbons, P. Guha, J. Math. Phys. 58, 032503 (2017). arXiv:1612.00375

10. S. Chanda, G.W. Gibbons, P. Guha, Int. J. Geom. Methods Mod. Phys. 14, 1730002 (2017). arXiv:1612.07395 
11. M. Szydlowski, M. Heller, W. Sasin, J. Math. Phys. 37, 346 (1996)

12. A.V. Tsiganov, The Maupertuis principle and canonical transformations of the extended phase space. J. Non Linear Math. Phys. 8, 157-182 (2001). arXiv:nlin/0101061

13. O.C. Pin, Curvature and mechanics. Adv. Math. 15, 269-311 (1975)

14. G.W. Gibbons, M.C. Werner, Class. Quant. Grav. 25, 235009 (2008). arXiv:0807.0854 [gr-qc]

15. G.W. Gibbons, C.M. Warnick, Phys. Rev. D 79, 064031 (2009). arXiv:0809.1571 [gr-qc]

16. G.W. Gibbons, C.A.R. Herdeiro, C.M. Warnick, M.C. Werner, Phys. Rev. D 79, 044022 (2009). arXiv:0811.2877 [gr-qc]
17. M.C. Werner, Gravitational lensing in the Kerr-Randers optical geometry. Gen. Relativ. Grav. 44, 3047 (2012)

18. A. Deriglazov, Classical Mechanics, Hamiltonian and Lagrangian Formalism (Springer, Heidelberg, Dordrecht, London, New York). https://doi.org/10.1007/978-3-642-14037-2

19. S. Chandrasekhar, The Mathematical Introduction of Black Holes (Oxford University Press, New York, 1983)

20. M. Olivares, J. Saavedra, C. Leiva, J.R. Villanueva, Mod. Phys. Lett. A 26, 2923 (2011). arXiv:1101.0748

21. G. Greenhill, Newton-Einstein planetary orbit. Philos. Mag. 41(6), 141-143 (1921) 